\title{
Effect of cell culture using chitosan membranes on stemness marker genes in mesenchymal stem cells
}

\author{
ZHIQIANG LI ${ }^{1}$, XIAOJUN TIAN ${ }^{2}$, YAN YUAN $^{2}$, ZHIXIU SONG $^{2}$, LILI ZHANG $^{3}$, \\ XIA WANG ${ }^{4}$ and TONG LI ${ }^{2}$
}

\begin{abstract}
Departments of ${ }^{1}$ Integrated Traditional Chinese and Western Medicine, ${ }^{2}$ Neurology, ${ }^{3}$ Gastroenterology and ${ }^{4}$ Tuberculosis, The First Affiliated Hospital of Xinxiang Medical University, Weihui, Henan 453100, P.R. China
\end{abstract}

Received November 30, 2012; Accepted April 4, 2013

DOI: $10.3892 / \mathrm{mmr} .2013 .1423$

\begin{abstract}
Mesenchymal stem cell (MSC) therapy is a promising treatment for diseases of the nervous system. However, MSCs often lose their stemness and homing abilities when cultured in conventional two-dimensional (2D) systems. Consequently, it is important to explore novel culture methods for MSC-based therapies in clinical practice. To investigate the effect of a cell culture using chitosan membranes on MSCs, the morphology of MSCs cultured using chitosan membranes was observed and the expression of stemness marker genes was analyzed. We demonstrated that MSCs cultured using chitosan membranes form spheroids. Additionally, the expression of stemness marker genes, including Oct4, Sox 2 and Nanog, increased significantly when MSCs were cultured using chitosan membranes compared with 2D culture systems. Finally, MSCs cultured using chitosan membranes were found to have an increased potential to differentiate into nerve cells and chrondrocytes. In conclusion, we demonstrated that MSCs cultured on chitosan membranes maintain their stemness and homing abilities. This finding may be further investigated for the development of novel cell-based therapies for diseases involving neuron-like cells and chondrogenesis.
\end{abstract}

\section{Introduction}

Stem cells may be used in the treatment of various diseases due to their differentiation activity and self-renewal potential. When subcultured over several generations, stem cells are unable to undergo differentiation or self-renewal, mainly due to the surrounding microenvironment, which is known to largely control the fate of stem cells. Recently, various biomaterials have been developed to maintain embryonic stem cell self-renewal. Mesenchymal stem cells (MSCs), one type of adult stem cell, are widely distributed in several tissues,

Correspondence to: Professor Tong Li, The First Affiliated Hospital of Xinxiang Medical University, 88 Jiankang Road, Weihui, Henan 453100, P.R. China

E-mail: tonglicn@163.com

Key words: chitosan, mesenchymal stem cell, stemness including bone marrow, cord blood and adipose tissue. MSCs are able to differentiate into a variety of cell types, including adipocytes, osteocytes, chondrocytes, myocytes and hepatocytes of the mesodermal lineage and nerve and epithelial cells of the ectodermal lineage (1-6). Besides differentiation, MSCs also release numerous cytokines to support tissue regeneration and repair, including insulin-like growth factor-1 (IGF-1), vascular endothelial growth factor- $\alpha$ (VEGF- $\alpha$ ) and macrophage chemokines (7). Numerous studies have shown that MSCs stimulate the regeneration and repair of damaged tissues, particularly in acute ischemic diseases, including acute myocardial or cerebral infarction and various types of arthritis $(1,3,5,6,8)$. Furthermore, previous studies have shown that MSCs have special immunological characteristics; therefore, MSCs may be transplanted without a rejection reaction $(9,10)$. In addition, MSCs are involved in immune regulation, thus they have the potential to be used as a novel therapy to treat rejection following organ transplantation and autoimmune diseases. Consequently, MSCs may have a therapeutic value in the treatment of numerous serious diseases and a broad scope in future clinical applications.

The regeneration, repair and immune regulation abilities of MSCs are largely due to their multi-lineage differentiation potential and paracrine actions. Currently, it is difficult to maintain the stability of MSCs under in vitro culture conditions, since aging, spontaneous differentiation into osteocytes and a decreased proliferation ability have been reported in MSC cultures under normal conditions (11-13). The spontaneous differentiation of MSCs decreases their ability to differentiate into other important cells, such as nerve and cardiac muscle cells. The transplantation of these differentiated MSCs (which are not morphologically similar to osteocytes) to non-bone tissues has been shown to result in ectopic calcification and the impairment of tissue functions (14). The paracrine actions of MSCs cultured in vitro have also been found to decrease and their myocardial protection ability was affected (15). Finally, the aging and differentiation of MSCs cultured in vitro had significant effects on their therapeutic outcome and safety.

Chitosan is a chitin derivative that is widely found in nature. It is composed of D-glucosamine and N-acetyl-glucosamine, which are joined by $\beta(1 \rightarrow 4)$ glycosidic bonds. Due to its biocompatibility, non-immunogenicity and non-toxicity, chitosan is compatible and cross-linked with collagen. The 
Table I. Primers used for quantitative real-time PCR (qRT-PCR).

\begin{tabular}{llr}
\hline Gene & \multicolumn{1}{c}{ Primer sequence } & Annealing temperature $\left({ }^{\circ} \mathrm{C}\right)$ \\
\hline$\beta$-actin & Sense: AACTACCTTCAACTCCATC & 55 \\
& Antisense: TGATCTTGATCTTCATTGTG & 60 \\
Oct4 & Sense: ACATCAAAGCTCTGCAGAAA & 58 \\
Sox2 & Antisense: CTGAATACCTTCCCAAATAGAAC & 60 \\
Nanog & Sense: TGCGAGCGCTGCACAT & 6 \\
& Antisense: GCAGCGTGTACTTATCCTTCTTCA & \\
\hline
\end{tabular}

different types of chitosan and its deacylation level have been found to affect cell adhesion and proliferation (16-19). Melanocytes and keratocytes cultured on chitosan-coated surfaces have been shown to form spheroids, which were maintained in three-dimensional (3D) culture systems $(16,20)$. In the present study, we investigated the effect of cell culture using chitosan membranes on the stemness of MSCs and provided information with regard to the clinical application of MSC-based therapy.

\section{Materials and methods}

Ethics. This study was conducted in accordance with the declaration of Helsinki. This study was conducted with approval from the Ethics Committee of the First Affiliated Hospital of Xinxiang Medical University, Weihui, China.

MSC culture. The first generation of bone marrow-derived human mesenchymal stem cells (hMSCs) were provided by the Shengzhen Baiwang Biotechnology Corporation (Shengzhen, China). hMSCs (100 cells $/ \mathrm{cm}^{2}$ ) were thawed and recovered for $24 \mathrm{~h}$, and then cultured in full medium (CCM) with $17 \%$ fetal bovine serum (FBS) for 7-8 days until 70\% fusion. hMSCs were passaged under the same conditions and were used in the experiment before the third generation.

Preparation of chitosan membranes. Chitosan powder (molecular weight, $510 \mathrm{kDa} ; 77.8 \%$ deacetylation; Shengzheng Baiwang Biotechnology Corporation) was dissolved in $1 \%$ aqueous acetic acid solution to obtain a $1 \%$ chitosan solution. Next, $300 \mu \mathrm{l}$ of the chitosan solution was coated on a glass coverslip and dried out at room temperature. Following neutralization with $0.5 \mathrm{~N} \mathrm{NaOH}$ for $30 \mathrm{~min}$, the glass coverslip was washed with PBS 5 times and exposed to ultraviolet radiation for $1 \mathrm{~h}$. The prepared chitosan membranes were used in further experiments.

Generation and dissociation of spheroids. hMSCs were cultured at a low density $(1,000$ cells/well) in 6-well plates containing chitosan membranes. Cell growth was observed daily using an inverted microscope. To obtain spheroid-derived cells, spheroids were incubated with trypsin/EDTA for 5-30 min (depending on the size of the spheroid). During dissociation, the cells were pipetted every 2-3 min.
Proliferation and survival ratio of MSCs cultured using chitosan membranes. The cell numbers of MSCs cultured with or without chitosan membranes were analyzed using the fluorescent dye Hoechst 33528 on days 1, 3 and 10 of culture. Spheroids and cells were digested in papain solution (Sigma, San Francisco, CA, USA) and then incubated with $0.1 \mathrm{mg} / \mathrm{ml}$ Hoechst 33528 dye (Sigma). The concentration was measured by a fluorescence spectrophotometer (F2500; Hitachi, Tokyo, Japan) at room temperature using an excitation and emission wavelength of 365 and $458 \mathrm{~nm}$, respectively. The cell number was calculated based on a standard curve and the cell activity was measured using a propidium iodide (PI) staining assay (Sigma Resources and Technologies, Inc., Santa Clara, CA, USA). PI solution $(2 \mathrm{mg} / \mathrm{ml})$ was added to the cell suspension and the percentage of unstained cells was defined as cell activity.

RNA extraction and quantitative real-time PCR ( $q R T-P C R)$ analysis. Total RNA was extracted from the cells using TRIzol reagent (Invitrogen, Carlsbad, CA, USA) according to the manufacturer's instructions. The isolated RNA was reverse transcribed and amplified using the RevertAid First Strand cDNA Synthesis Kit (MBI Fermentas, St. Leon-Rot, Germany). cDNA was then used as a template for qRT-PCR to quantitatively analyze the gene expression levels with the DyNAmo Flash SYBR Green qPCR kit (Finnzymes, Espoo, Finland). $\beta$-actin was amplified and used as an internal standard to normalize the expression of tested genes. The primers of the genes investigated are listed in Table I.

MSC differentiation into nerve cells. MSCs were primarily cultured for 3 days to induce differentiation into nerve cells. Following the addition of $10 \mathrm{ng} / \mathrm{ml}$ epidermal growth factor (EGF; Gibco-BRL, Rockville, MD, USA), 20 ng/ml hepatocyte growth factor (HGF; R\&D Systems, Minneapolis, MN, USA) and $20 \mathrm{ng} / \mathrm{ml}$ VEGF (R\&D Systems) were added to the culture medium, and the cells were cultured for an additional 10 days.

Induction of chondrogenic differentiation. To induce chondrogenic differentiation, basic medium was replaced by chondrogenic differentiation medium following a primary culture for 3 days. Chondrogenic differentiation medium contained $10 \mathrm{ng} / \mathrm{ml}$ transforming growth factor $\beta 3$ (TGF $\beta 3$ ) 


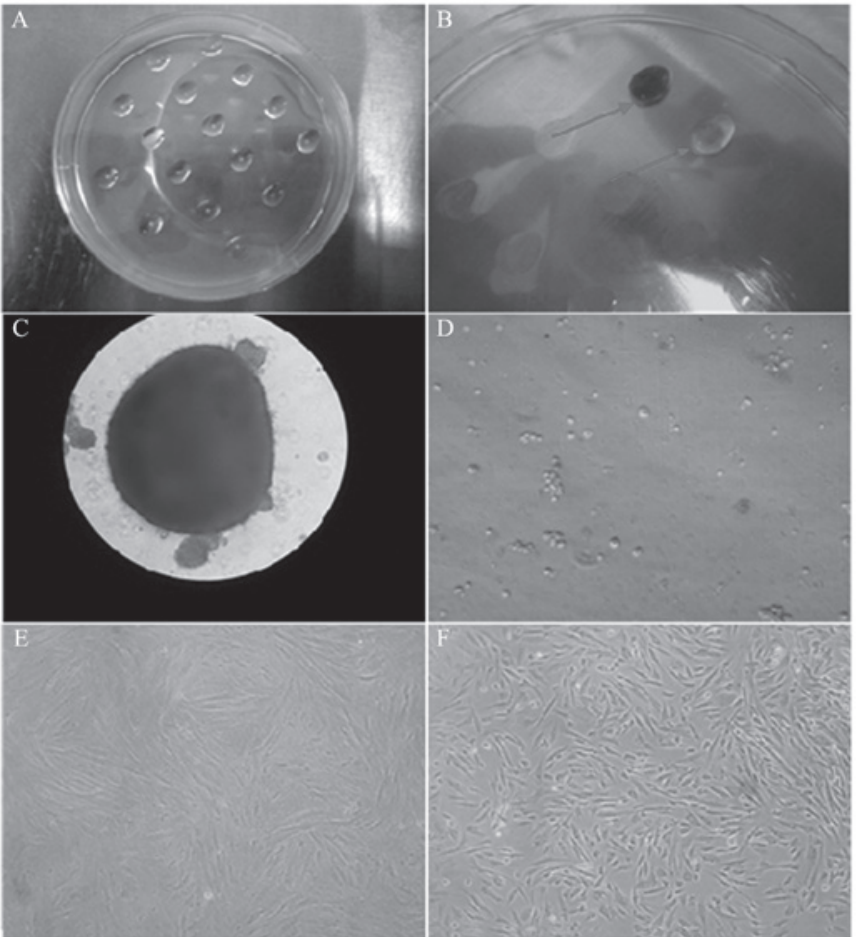

Figure 1. Effect of cell culture using chitosan membranes on MSC morphology. (A) Mode of colony formation following cell culture using chitosan membranes; (B) chitosan membrane culture was stained with trypan blue; (C) observation of spheroid formation following cell culture using chitosan membranes under a microscope; (D) the spheroid number was calculated following digestion with $0.25 \%$ trypsin and staining with trypan blue ( $\mathrm{x} 400)$; (E) P7 under general adherent culture (x400); (F) P7 under adherent culture following culture using chitosan membranes (x400). MSC, mesenchymal stem cell. P7, seventh passage cells.

(CytoLab/PeproTech, Rehovot, Israel), $0.1 \mathrm{mM}$ dexamethasone, $50 \mathrm{mg} / \mathrm{ml} \mathrm{L}$-ascorbic acid 2-phosphate, $40 \mathrm{mg} / \mathrm{ml}$ L-proline, $1 \%$ insulin transferrin selenium (ITS-premix 100; Sigma), $1 \%$ penicillin and $1 \%$ streptomycin. The medium was replaced twice a week. For morphological observation, the cells were incubated in precooled acetone and washed with PBS. The cells were then stained with Mayer's hematoxylin solution and washed once with toluidine blue and water. Safranin O was then applied for $5 \mathrm{~min}$ to stain the cells. Subsequently, 95\% alcohol, absolute alcohol and xylene were sequentially used to treat the cells once every $2 \mathrm{~min}$. Finally, differentiation was observed under a microscope.

Statistical analysis. The data were analyzed using SPSS 12.0.1 software and are presented as the mean \pm standard error (SE). $\mathrm{P}<0.05$ was considered to indicate a statistically significant difference.

\section{Results}

Morphology of MSCs cultured using chitosan membranes. MSCs cultured in a 2D culture system had a typical long polygonal morphology. However, spheroid formation was observed within 1-2 days when the cells were transferred to chitosan membranes. Cell size was reduced by $75 \%$ compared with cells cultured in a 2D system following trypsin digestion, similar to spheroids generated using the pendant drop method (Fig. 1).

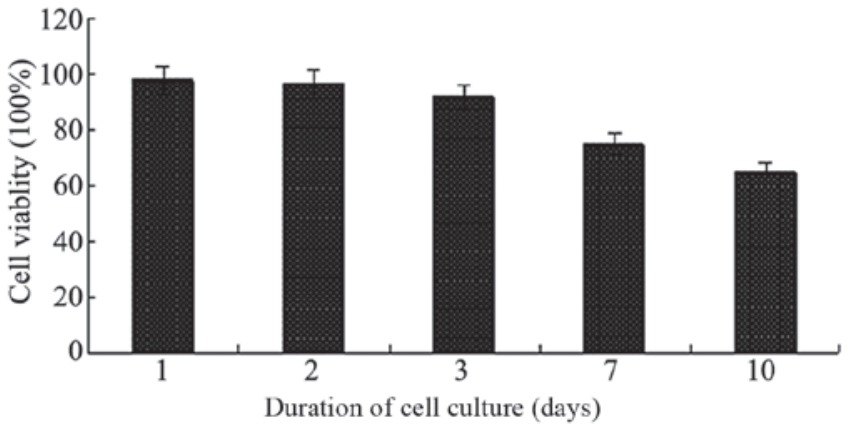

Figure 2. Survival percentage of MSCs following cell culture using chitosan membranes. The survival percentage was $>90 \%$ during the first 3 days of culture and the cell survival ratio remained $>65 \%$ after 10 days of culture. MSC, mesenchymal stem cell.

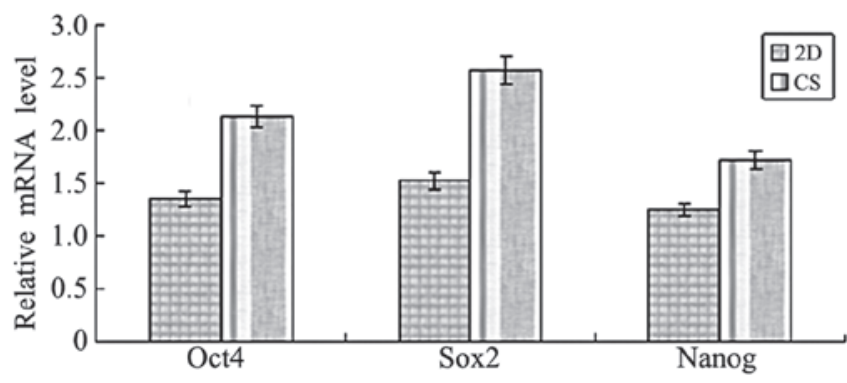

Figure 3. Gene expression levels of stemness marker genes Oct4, Sox 2 and Nanog in MSCs following cell culture using chitosan membranes. Each experiment was repeated 3 times and the relative RNA level is presented as the mRNA ratio of each gene compared with GAPDH. CS, chitosan membrane cultures; MSC, mesenchymal stem cell; 2D, two-dimensional.

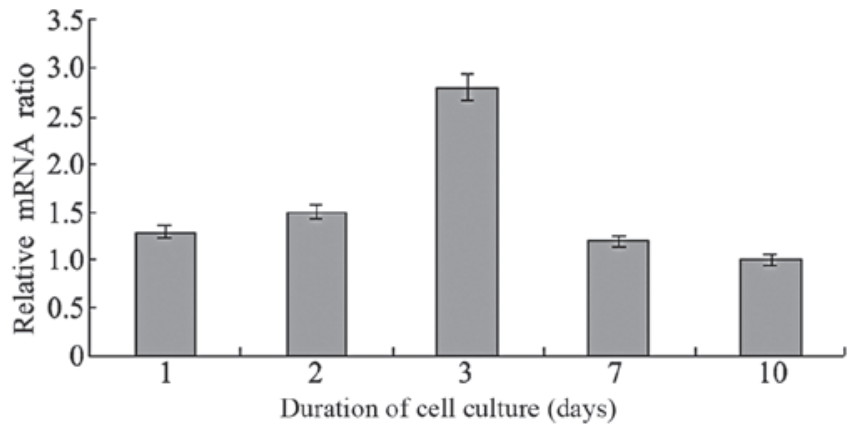

Figure 4. Relative Sox 2 mRNA ratios of MSCs cultured using chitosan membranes compared with 2D culture on days 1, 2, 3, 7 and 10 of cell culture. The expression level of Sox 2 was maintained when MSCs were cultured using chitosan membranes. MSC, mesenchymal stem cell; 2D, two-dimensional.

Spheroid cell viability. Since MSC spheroids have limited access to nutritional agents, cell viability was investigated. The 10,000 or 25,000 MSC spheroid was cultured for 3 days and $90 \%$ of harvested cells survived. The number of apoptotic cells slightly increased with the duration of culture (Fig. 2). Growth curves for cultured MSCs were plotted for the different culture materials used. MSC proliferation was found to be maintained after spheroid formation.

Effects of spheroid formation on the expression of stemness marker genes. To determine the regulatory roles of stemness 


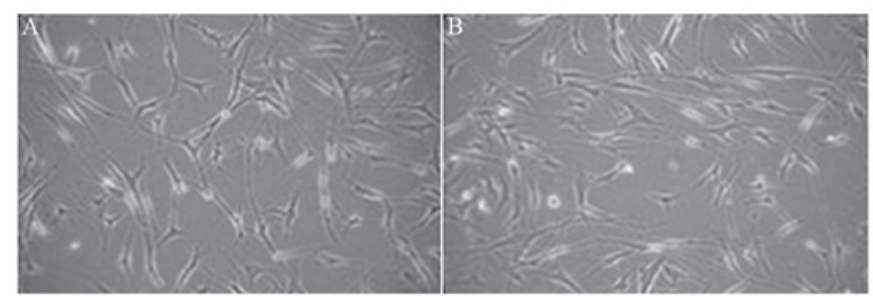

Figure 5. MSCs were cultured using chitosan membranes for 3 days and then transfered to a 2D culture system. The ability of the cells to differentiate into nerve cells in the two culture systems was compared. (A) MSCs cultured in a 2D culture system for 3 days (x400); (B) MSCs cultured using chitosan membranes for 3 days (x400). MSC, mesenchymal stem cell; 2D, two-dimensional.

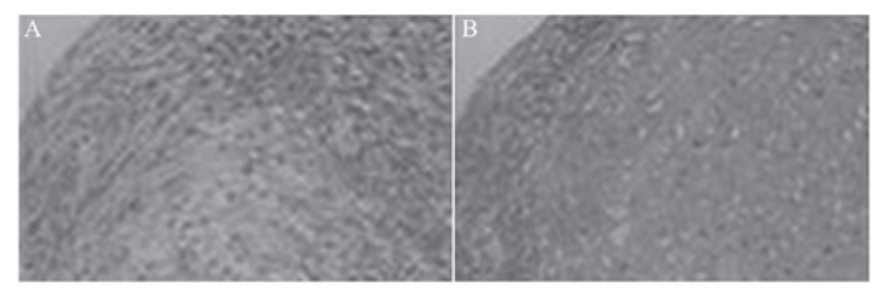

Figure 6. Ability of MSCs to differentiate into chondro metrocytes following treatment with TGF $\beta 3$. (A) MSCs cultured in a $2 \mathrm{D}$ culture system (x400); (B) MSCs cultured using chitosan membranes, followed by transfer to a $2 \mathrm{D}$ culture system (x400). MSC, mesenchymal stem cell; 2D, two-dimensional TGF $\beta 3$, transforming growth factor $\beta 3$.

marker genes in MSC culture, the expression levels of Oct4, Sox 2 and Nanog mRNA with different culture materials were analyzed by RT-PCR (Figs. 3 and 4). A ratio $>1$ was considered to indicate upregulated gene expression, while $<1$ showed downregulation. The expression levels of these genes in MSCs cultured using chitosan membranes were upregulated within 1-3 days, and downregulated after 7 days. This indicated that spheroid formation by MSCs cultured using chitosan membranes may be able to maintain the expression of MSC stemness marker genes.

Effect of culture using chitosan membranes on MSC differentiation. Cells cultured in medium supplemented with growth factors were differentiated and transformed into cells with a long and thin morphology. The change in cell morphology was similar to that of nerve cells; for example, the cytoplasmic area around the nucleolus was smaller (Fig. 5). MSCs cultured using chitosan membranes had an increased differentiation efficiency $(\mathrm{P}<0.001)$. These results indicated that MSCs were able to differentiate into nerve cells in the presence of these growth factors, and that the MSCs cultured on chitosan membranes had an increased potential for this differentiation. When TGF 33 was added to the medium of MSCs cultured using chitosan membranes, cells differentiated into cartilage after 2 weeks, and chitosan membranes were found to aid this type of differentiation (Fig. 6). These results indicated that spheroids formed by MSCs cultured using chitosan membranes were similar to MSC spheroids generated by the pendant drop method. This method maintained the stemness ability of MSCs and may aid the induction of differentiation under specific conditions.

\section{Discussion}

Stem cells are known for their self-renewal ability and may be induced to differentiate into specific cell types when cultured under particular conditions. They are also considered to be important candidates for tissue engineering. However, pluripotent stem cells lose their stemness ability when cultured in vitro. To maintain the self-renewal ability of undifferentiated embryonic stem cells (ESCs), the feeder layer, such as mouse embryonic fibroblasts (MEFs) or conditional medium, is required to provide a stable environment $(21,22)$. Numerous natural or synthetic polymeric materials have been developed to replace the feeder layer in order to maintain the self-renewal ability of ESCs (23-27). Transcription factors Oct 4 , Sox 2 and Nanog have been shown to be important in the maintenance of pluripotency in ESCs $(28,29)$. These marker genes are also expressed in adult stem cells $(30,31)$. In the present study, we investigated the effect of cell culture using chitosan membranes on the development and self-renewal ability of undifferentiated MSCs. MSCs were demonstrated to rapidly form 3D spheroids following culture using chitosan membranes, and the expression levels of marker genes (Oct4, Sox 2 and Nanog) either remained stable or were upregulated.

3D multicellular states have been previously described in a number of cell types, including ESC-derived embryoid bodies and neurosphere-derived neurons (32). This information was useful for the development of ESCs. MSCs have been shown to form 3D spheroids when cultured using micropore membranes (33) or other types of surfaces $(34,35)$. Human MSCs cultured using micropore membranes formed spheroids and exhibited increased anti-inflammatory properties in a previous study (35). Spheroid formation following 2D cell culture and the expression of differentiation-related genes have not previously been investigated. In the present study, the increased expression of stemness-related genes indicated an improved plasticity of MSCs. The accumulation of MSCs created a suitable environment for cell-cell interactions, and promoted the early differentiation of MSCs into nerve cells and chondrocytes (36). Various natural and synthetic 3D scaffolds have been demonstrated to be beneficial for the formation of nerve cells and cartilage $(37,38)$. The spheroid formation of MSCs that occurred during culture using chitosan membranes not only enhanced cellular plasticity, but also improved nerve cell and cartilage formation. The disadvantage of 3D multicellular status may be the finite spreading of center. However, our results indicated that the cell survival ratio was $>70 \%$, even 7 days after spheroid formation, and that $\geq 90 \%$ cells had survived 3 days after spheroid formation.

In conclusion, 3D MSC spheroids were observed in 2D cultures within 1-2 days when using chitosan membranes. The expression levels of stemness marker genes remained stable in these spheroids; however, decreased expression levels of stemness marker genes suppressed spheroid formation. These results indicate that undifferentiated MSCs are able maintain their original state while forming 3D spheroids when cultured using chitosan membranes. Furthermore, spheroids exhibited improved nerve cell formation and chondrogenic differentiation abilities following induction. 3D spheroid formation on biomaterials may constitute a novel strategy to maintain the self-renewal ability of MSCs and increase the differentiation 
potential of MSCs into cartilage, and has the potential to be applied in the treatment of diseases of the nervous system and cartilage tissue engineering. However, the underlying mechanism regulating spheroid formation requires further investigation.

\section{References}

1. Phinney DG and Prockop DJ: Concise review: mesenchymal stem/multipotent stromal cells: the state of transdifferentiation and modes of tissue repair - current views. Stem Cells 25: 2896-2902, 2007.

2. Pittenger MF, Mackay AM, Beck SC, et al: Multilineage potential of adult human mesenchymal stem cells. Science 284 143-147, 1999

3. Pittenger MF and Martin BJ: Mesenchymal stem cells and their potential as cardiac therapeutics. Circ Res 95: 9-20, 2004

4. Prockop DJ: Marrow stromal cells as stem cells for nonhematopoietic tissues. Science 276: 71-74, 1997.

5. Wu Y, Chen L, Scott PG and Tredget EE: Mesenchymal stem cells enhance wound healing through differentiation and angiogenesis. Stem Cells 25: 2648-2659, 2007.

6. Wu Y, Zhao RC and Tredget EE: Concise review: bone marrow-derived stem/progenitor cells in cutaneous repair and regeneration. Stem Cells 28: 905-915, 2010.

7. Chen L, Tredget EE, Wu PY and Wu Y: Paracrine factors of mesenchymal stem cells recruit macrophages and endothelial lineage cells and enhance wound healing. PLoS One 3: e1886, 2008.

8. Nagai A, Kim WK, Lee HJ, et al: Multilineage potential of stable human mesenchymal stem cell line derived from fetal marrow. PLoS One 2: e1272, 2007.

9. Aggarwal S and Pittenger MF: Human mesenchymal stem cells modulate allogeneic immune cell responses. Blood 105 $1815-1822,2005$.

10. Jiang XX, Zhang Y, Liu B, Zhang SX, Wu Y, Yu XD and Mao N: Human mesenchymal stem cells inhibit differentiation and function of monocyte-derived dendritic cells. Blood 105: 4120-4126, 2005.

11. Baxter MA, Wynn RF, Jowitt SN, Wraith JE, Fairbairn LJ and Bellantuono I: Study of telomere length reveals rapid aging of human marrow stromal cells following in vitro expansion. Stem Cells 22: 675-682, 2004.

12. Krampera M, Pasini A, Rigo A, et al: HB-EGF/HER-1 signaling in bone marrow mesenchymal stem cells: inducing cell expansion and reversibly preventing multilineage differentiation. Blood 106: 59-66, 2005.

13. Wagner W, Horn P, Castoldi M, et al: Replicative senescence of mesenchymal stem cells: a continuous and organized process. PLoS One 3: e2213, 2008.

14. Pelttari K, Winter A, Steck E, et al: Premature induction of hypertrophy during in vitro chondrogenesis of human mesenchymal stem cells correlates with calcification and vascular invasion after ectopic transplantation in SCID mice. Arthritis Rheum 54: 3254-3266, 2006.

15. Crisostomo PR, Wang M, Wairiuko GM, Morrell ED, Terrell AM, Seshadri P, Nam UH and Meldrum DR: High passage number of stem cells adversely affects stem cell activation and myocardial protection. Shock 26: 575-580, 2006.

16. Lin SJ, Jee SH, Hsaio WC, Lee SJ and Young TH: Formation of melanocyte spheroids on the chitosan-coated surface. Biomaterials 26: 1413-1422, 2005.

17. Yang TL and Young TH: The specificity of chitosan in promoting branching morphogenesis of progenitor salivary tissue. Biochem Biophys Res Commun 381: 466-470, 2009.

18. Yeh LK, Chen YH, Chiu CS, Hu FR, Young TH and Wang IJ: The phenotype of bovine corneal epithelial cells on chitosan membrane. J Biomed Mater Res A 90: 18-26, 2009.

19. Chatelet C, Damour O and Domard A: Influence of the degree of acetylation on some biological properties of chitosan films Biomaterials 22: 261-268, 2001.
20. Chen YH, Wang IJ and Young TH: Formation of keratocyte spheroids on chitosan-coated surface can maintain keratocyte phenotypes. Tissue Eng Part A 15: 2001-2013, 2009.

21. Chiao E, Kmet M, Behr B and Baker J: Derivation of human embryonic stem cells in standard and chemically defined conditions. Methods Cell Biol 86: 1-14, 2008

22. Richards M, Fong CY, Chan WK, Wong PC and Bongso A: Human feeders support prolonged undifferentiated growth of human inner cell masses and embryonic stem cells. Nat Biotechnol 20: 933-936, 2002.

23. Lee ST, Yun JI, Jo YS, et al: Engineering integrin signaling for promoting embryonic stem cell self-renewal in a precisely defined niche. Biomaterials 31: 1219-1226, 2010.

24. Brafman DA, Chang CW, Fernandez A, Willert K, Varghese S and Chien S: Long-term human pluripotent stem cell self-renewal on synthetic polymer surfaces. Biomaterials 31: 9135-9144, 2010.

25. Nur-E-Kamal A, Ahmed I, Kamal J, Schindler M and Meiners S: Three-dimensional nanofibrillar surfaces promote self-renewal in mouse embryonic stem cells. Stem Cells 24: 426-433, 2006.

26. Gerecht S, Burdick JA, Ferreira LS, Townsend SA, Langer R and Vunjak-Novakovic G: Hyaluronic acid hydrogel for controlled self-renewal and differentiation of human embryonic stem cells. Proc Natl Acad Sci USA 104: 11298-11303, 2007.

27. Abraham S, Eroshenko N and Rao RR: Role of bioinspired polymers in determination of pluripotent stem cell fate. Regen Med 4: 561-578, 2009.

28. Kopp JL, Ormsbee BD, Desler M and Rizzino A: Small increases in the level of Sox2 trigger the differentiation of mouse embryonic stem cells. Stem Cells 26: 903-911, 2008.

29. Sokolov MV, Panyutin IV, Onyshchenko MI, Panyutin IG and Neumann RD: Expression of pluripotency-associated genes in the surviving fraction of cultured human embryonic stem cells is not significantly affected by ionizing radiation. Gene 455: 8-15, 2010.

30. Baer PC, Griesche N, Luttmann W, Schubert R, Luttmann A and Geiger H: Human adipose-derived mesenchymal stem cells in vitro: evaluation of an optimal expansion medium preserving stemness. Cytotherapy 12: 96-106, 2010.

31. Fortunel NO, Otu HH, Ng HH, et al: Comment on 'Stemness: transcriptional profiling of embryonic and adult stem cells' and 'a stem cell molecular signature'. Science 302: 393, 2003.

32. Kim SY and Im GI: Control of the chondrogenesis during the development and its application for cartilage tissue engineering. J Tissue Eng Regen Med 6: 595-602, 2009.

33. Bartosh TJ, Ylöstalo JH, Mohammadipoor A, et al: Aggregation of human mesenchymal stromal cells (MSCs) into 3D spheroids enhances their antiinflammatory properties. Proc Natl Acad Sci USA 107: 13724-13729, 2010

34. Wang W, Itaka K, Ohba S, Nishiyama N, Chung UI, Yamasaki Y and Kataoka K: 3D spheroid culture system on micropatterned substrates for improved differentiation efficiency of multipotent mesenchymal stem cells. Biomaterials 30: 2705-2715, 2009.

35. Miyagawa Y, Okita H, Hiroyama M, et al: A microfabricated scaffold induces the spheroid formation of human bone marrow-derived mesenchymal progenitor cells and promotes efficient adipogenic differentiation. Tissue Eng Part A 17: 513-521, 2011.

36. Quintana L, zur Nieden NI and Semino CE: Morphogenetic and regulatory mechanisms during developmental chondrogenesis: new paradigms for cartilage tissue engineering. Tissue Eng Part B Rev 15: 29-41, 2009.

37. Chang JC, Hsu SH and Chen DC: The promotion of chondrogenesis in adipose-derived adult stem cells by an RGD-chimeric protein in 3D alginate culture. Biomaterials 30: 6265-6275, 2009.

38. Martinez-Diaz S, Garcia-Giralt N, Lebourg M, et al: In vivo evaluation of 3-dimensional polycaprolactone scaffolds for cartilage repair in rabbits. Am J Sports Med 38: 509-519, 2010. 